\title{
Hadronic Production of Colored SUSY Particles with Electroweak NLO Contributions
}

\author{
Wolfgang Hollik, Monika Kollar, Edoardo Mirabella and Maike K. Trenkel
}

\author{
Max-Planck-Institut für Physik, Föhringer Ring 6, D-80805 München, Germany
}

\begin{abstract}
We consider the production of squarks and gluinos at hadronic colliders. An overview over the class of processes is given. We investigate in detail the tree-level and higher order EW contributions to the cross sections. Special care has to be taken to obtain infrared finite observables. We study numerically stop-anti-stop and squark-gluino production at the LHC.
\end{abstract}

Keywords: Supersymmetric Standard Model, NLO Computations, Hadronic Colliders

PACS: $12.15 . \mathrm{Lk}, 14.80 \mathrm{Ly}$

\section{INTRODUCTION}

If SUSY is realized, colored SUSY particles will provide large production cross sections at hadron colliders since they are produced via the strong interaction. Pair production of squarks and gluinos is, therefore, among the most promising SUSY discovery channels.

The cross sections are substantially altered by higher order corrections. NLO QCD corrections at $\mathscr{O}\left(\alpha_{s}^{3}\right)$ range typically at $20-30 \%$ [1] or even larger values for topsquark pair production [2]. It was shown that they reduce the factorization and renormalization scale dependence considerably. Only recently also NLO EW corrections of $\mathscr{O}\left(\alpha_{s}^{2} \alpha\right)$ were considered, to top-squark pair production $[3,4]$, to pair production of squarks of the first generations [5], and to squark-gluino production [6].

The aim of this talk is to provide an overview over the class of processes and to illustrate the effects of the EW contributions. The focus will be on top-squarks (stops $\tilde{t}$ ), candidates for the lightest squark within many SUSY models owing to the large top-Yukawa coupling [7], and on squark-gluino ( $\tilde{q} \tilde{g})$ final states, constituting an important fraction of colored SUSY particle production processes in a wide range of the parameter space. We investigate in detail the various EW contributions to the cross sections, including tree-level $\mathscr{O}\left(\alpha^{2}+\alpha_{s} \alpha\right)$ and NLO $\mathscr{O}\left(\alpha_{s}^{2} \alpha\right)$ corrections. Special care has to be taken to obtain infrared (IR) finite observables. We present numerical results for $\tilde{t} \tilde{t}^{*}$ and $\tilde{q} \tilde{g}$ production within the specific SUSY scenario SPS1a' [8].

\section{TREE-LEVEL CONTRIBUTIONS}

At hadron colliders, pair production of squarks and gluinos proceeds at lowest oder QCD via the following partonic processes.

- Gluino pairs are produced by $g g$ and $q \bar{q}$ initial states.
- Squark-gluino final states require quark-gluon initial states, the quark and the produced squark being of the same flavor. Accordingly, $\tilde{t} \tilde{g}$ final states cannot be produced at LO owing to the vanishing top-quark PDF, and $\tilde{b} \tilde{g}$ production is suppressed. Furthermore, bottomsquarks are experimentally distinguishable from other squarks by their decay products. We therefore restrict the discussion of $\tilde{q} \tilde{g}$ production to left- and right-handed (anti-)squarks of the first two generations.

- Squark-anti-squark pairs are produced via $g g$ fusion and $q \bar{q}$ annihilation. The $q \bar{q}$ initiated processes can be either gluon-mediated $s$-channel diagrams (with quarks of any flavor in the initial state) or gluino-mediated $t$ channel diagrams. The latter also allow for $\tilde{q} \tilde{q}$ production, which will be discussed elsewhere.

- Stop-anti-stop (and sbottom-anti-sbottom) pair production has to be discussed separately from $\tilde{q} \tilde{q}^{*}$ production since, excluded by the PDF, it does not proceed via $t$-channel diagrams. Moreover, squarks of the third generation are experimentally distinguishable and the L-Rmixing has to be taken into account. Due to the absence of the $t$-channel diagram, stops (and sbottoms) can only be produced diagonally at LO.

These production mechanisms of $\mathscr{O}\left(\alpha_{s}^{2}\right)$ are the dominant ones for squark and gluino production at hadronic colliders. Besides, diagonal and non-diagonal squark pairs can also be produced by $q \bar{q}$ induced tree-level EW processes $[9,10]$. For stops and sbottoms, only $s$-channel diagrams with $\gamma$ or $Z$ exchange are present at $\mathscr{O}\left(\alpha^{2}\right)$ and give negligible contributions. However for squarks of the first generations also $t$-channel diagrams mediated by $\tilde{\chi}^{0}$ or $\tilde{\chi}^{ \pm}$exchange contribute and, additionally, non-zero interferences of $\mathscr{O}\left(\alpha_{s} \alpha\right)$ between the QCD and EW diagrams arise.

Finally, photon-induced processes are further treelevel channels that contribute to (top-)squark pair production (via photon-gluon fusion) and to squark-gluino 
production (via photon-quark fusion). Although these channels are in general suppressed by the photon distribution in the proton which is only non-zero at NLO QED, they can become sizable as pointed out in $[3,5]$.

\section{EW NLO CORRECTIONS}

One subset of EW NLO corrections are the virtual corrections. They arise from the interference between treelevel QCD diagrams and diagrams with one-loop EW insertions. In case of $q \bar{q}$ initial states, further $\mathscr{O}\left(\alpha_{s}^{2} \alpha\right)$ corrections arise from the interference of $\mathscr{O}(\alpha)$ EW treelevel and $\mathscr{O}\left(\alpha_{s}^{2}\right) \mathrm{QCD}$ box diagrams.

Getting an UV finite result requires renormalization of the involved quarks and squarks, which has been performed in the on-shell scheme. In case of $\tilde{t} \tilde{t}^{*}$ production, it is not necessary to renormalize the gluon field and the strong coupling $\alpha_{s}$ at this order of perturbation theory. Similarly for $\tilde{q} \tilde{g}$ production, also the gluino field needs not to be renormalized. The situation is more involved for $\tilde{q} \tilde{q}^{*}$ production, where the full one-loop QCD amplitude enters through interference contributions with the (enlarged) EW tree-level diagrams [5].

As a second type of singularities, one has to deal with IR (soft) singularities that arise from loop diagrams involving virtual photons. An IR finite result is obtained only in the sum of virtual corrections and real photon radiation processes. The diagrams are regularized by introducing a fictitious photon mass. For $\tilde{q} \tilde{q}^{*}$ and $\tilde{t} \tilde{t}^{*}$ production, also virtual gluons arise and become singular in the soft limit. To obtain an IR finite result, real gluon radiation processes have to be included at the appropriate order. The gluonic IR singularities are Abelian-like and can similarly be regularized by a finite gluon mass.

In case of a light quark in the initial state, also collinear singularities occur if the quark radiates off a photon in the collinear limit. We therefore keep the initial state quark masses as regulators where necessary. Parts of the singularities drop out in the sum of virtual and real corrections. Single logarithms owing to collinear singularities, however, survive and have to be absorbed into the quark parton distribution functions (PDFs). This can formally be achieved by a redefinition of the PDFs at NLO QED, in complete analogy to factorization for NLO QCD calculations [11, 12].

As mentioned before, $\tilde{t} \tilde{t}^{*}$ production at $\mathscr{O}\left(\alpha_{s}^{2} \alpha\right)$ also includes interference contributions from tree-level EW diagrams and one-loop QCD boxes. Providing an additional source of soft gluon singularities, these terms are essential in the sum of virtual and real gluon corrections in order to obtain an IR finite result. In case of $\tilde{q} \tilde{q}^{*}$ production the real gluon radiation also exhibits collinear singularities and the redefinition of the PDFs has to be done accordingly.
TABLE 1. Integrated cross sections for $\tilde{t}_{1} \tilde{t}_{1}^{*}$ and $\tilde{q} \tilde{g}$ production at the LHC within the SPS1a ${ }^{\prime}$ scenario.

\begin{tabular}{ccccc}
\hline final state & $\sigma^{\mathrm{LO}}$ & $\sigma_{\alpha_{s}^{2} \alpha}^{\mathrm{EW}}$ & $\sigma_{\alpha_{s} \alpha}^{\mathrm{EW}}$ & $\delta^{\mathrm{EW}}$ \\
\hline$\tilde{t}_{1} \tilde{t}_{1}^{*}$ & $1830 \mathrm{fb}$ & $-15.0 \mathrm{fb}$ & $34.1 \mathrm{fb}$ & $1.0 \%$ \\
\hline$\tilde{u}_{R} \tilde{g}+\tilde{d}_{R} \tilde{g}$ & $8900 \mathrm{fb}$ & $14.6 \mathrm{fb}$ & $5.05 \mathrm{fb}$ & $0.22 \%$ \\
$\tilde{u}_{L} \tilde{g}+\tilde{d}_{L} \tilde{g}$ & $8220 \mathrm{fb}$ & $-197 \mathrm{fb}$ & $4.62 \mathrm{fb}$ & $-2.3 \%$ \\
$\tilde{q} \tilde{g}$ & $17120 \mathrm{fb}$ & $-183 \mathrm{fb}$ & $9.67 \mathrm{fb}$ & $-1.0 \%$ \\
\hline
\end{tabular}

Finally, real (anti-)quark radiation processes give contributions of $\mathscr{O}\left(\alpha_{s}^{2} \alpha\right)$ through the interference of EW mediated and QCD mediated diagrams. For $\tilde{t}^{*}$ and $\tilde{q} \tilde{g}$ production, they are IR and collinear finite. In some diagrams, internal-state SUSY particles can go on-shell and the propagators are regularized by inserting the width of the respective particle. For $\tilde{t} \tilde{t}^{*}$ production, however, only SM particles appear as internal particles and real quark radiation is negligible and, therefore, not included in the numerical analysis.

\section{NUMERICAL RESULTS}

For the numerical discussion we refer to the mSUGRA scenario SPS1a ${ }^{\prime}$ [8] and use Soft susy [13] to evolve the GUT scale parameters down to lower scales. Factorization and renormalization scale are set equal to $1 \mathrm{TeV}$. A translation of the $\overline{\mathrm{DR}}$ parameters into the on-shell scheme is achieved by relating the renormalized masses at the one-loop level, leading to $m\left(\tilde{t}_{1}\right)=359.6 \mathrm{GeV}$ as the mass of the lighter stop. The top-quark mass is set to $170.9 \mathrm{GeV}$, all other Standard Model parameters are chosen in accordance with [8]. As discussed above, we need a set of PDFs that includes NLO QED corrections, as provided by MRST 2004 QED [14].

In Table 1 we present the integrated cross sections for $\tilde{t}_{1} \tilde{t}_{1}^{*}$ and $\tilde{q} \tilde{g}$ production at the LHC. Given are the LO, the $\mathscr{O}\left(\alpha_{s}^{2} \alpha\right)$, and the photon-induced $\mathscr{O}\left(\alpha_{s} \alpha\right)$ cross sections, and the complete EW contribution relative to the $\mathrm{LO}$ result, in obvious notation. For $\tilde{t}_{1} \tilde{t}_{1}^{*}$ production, the photon-induced processes dominate over the NLO $\mathscr{O}\left(\alpha_{s}^{2} \alpha\right)$ corrections and change the result substantially. The complete EW contribution is small and positive (1\%). For $\tilde{q} \tilde{g}$ final states, we present cross sections for left-handed and right-handed squarks separately and also give the inclusive results. Squarks of the second generation and charge conjugated processes are summed over, differing only by the required PDF. The photon-induced channels are less important than for stops, mainly suppressed by the parton density of the initial quark which is rather sensitive to the high final state masses $\left[m\left(\tilde{u}_{R}\right)=543.4 \mathrm{GeV}, m\left(\tilde{u}_{L}\right)=560.7 \mathrm{GeV}\right.$, $m\left(\tilde{d}_{R}\right)=539.4 \mathrm{GeV}, \quad m\left(\tilde{d}_{L}\right)=566.4 \mathrm{GeV}, \quad m(\tilde{g})=$ 

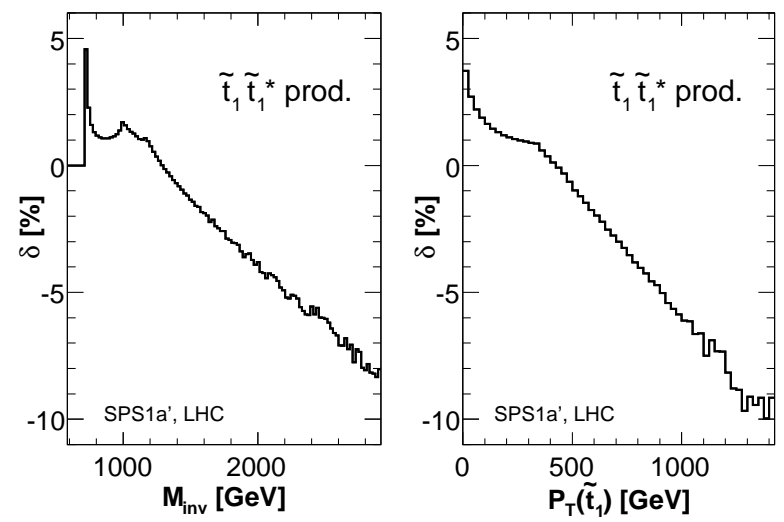

FIGURE 1. EW contribution relative to the LO cross section for $\tilde{t}_{1} \tilde{t}_{1}^{*}$ production at the LHC. Shown are the distributions with respect to the invariant mass of the $\tilde{t}_{1} \tilde{t}_{1}^{*}$ pair and to the transverse momentum of one of the stops.

$609.0 \mathrm{GeV}]$. Note that $\sigma_{\alpha_{s} \alpha}^{\mathrm{EW}}$ is independent on the chirality of the produced squark. Virtual and real corrections, of course, are not and $\sigma_{\alpha_{s}^{2} \alpha}^{\mathrm{EW}}$ is only important for left-handed squarks. The total EW contribution amounts $-1 \%$, for inclusive $\tilde{q} \tilde{g}$ production.

We illustrate the numerical impact of the EW contribution on the LO cross section in Fig. 1 and Fig. 2.

Fig. 1 refers to $\tilde{t}_{1} \tilde{t}_{1}^{*}$ production and shows the relative EW contribution as distribution with respect to the invariant mass $\left(M_{\text {inv }}\right)$ of the $\tilde{t}_{1} \tilde{t}_{1}^{*}$ pair and to the transverse momentum ( $\left.p_{T}\right)$ of one of the stops. In the $M_{\text {inv }}$ distribution, threshold effects arise from stop and sbottom pairs in the loop diagrams. In total, the EW contributions grow in size for increasing $M_{\mathrm{inv}}$ and $p_{T}$ and reach about $-10 \%$ for $M_{\mathrm{inv}} \sim 3 \mathrm{TeV}$ and $p_{T} \sim 1.5 \mathrm{TeV}$.

Fig. 2 refers to $\tilde{q} \tilde{g}$ production. Again, we consider left- and right-handed squarks separately and give also the inclusive results. Presented is the relative EW contribution with respect to $p_{T}$ of the produced gluino. Differences for $p_{T}(\tilde{q})$ are expected to be small, only arising from real photon and real quark radiation processes. For left-handed squarks, the EW contribution reaches the $10 \%$ level in the high- $p_{T}$ range. It is negligible, however, for right-handed squarks and the distribution is almost flat. As a consequence, the EW contribution to inclusive $\tilde{q} \tilde{g}$ production is moderate, about $-5 \%$ for $p_{T} \sim 1.5 \mathrm{TeV}$.

\section{CONCLUSIONS}

We considered the hadronic production of colored SUSY particles, squarks and gluinos, and classified the various processes. We focused on the EW contribution, due to tree-level $\mathscr{O}\left(\alpha_{s} \alpha\right)$ processes and NLO $\mathscr{O}\left(\alpha_{s}^{2} \alpha\right)$ corrections. We numerically investigated $\tilde{t}_{1} \tilde{t}_{1}^{*}$ and $\tilde{q} \tilde{g}$ production

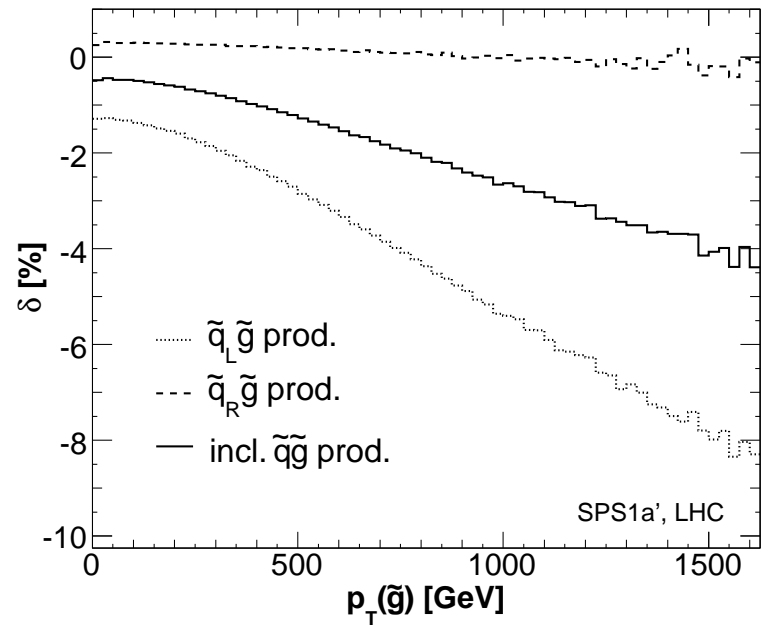

FIGURE 2. EW contribution relative to the LO cross section for $\tilde{q} \tilde{g}$ production at the LHC as distribution with respect to the transverse momentum of the gluino.

at the LHC within the SPS1a ${ }^{\prime}$ scenario. The EW contribution to the total cross sections is small, however it reaches (in size) the $10-20 \%$ level in the $p_{T}$ and invariant mass distributions and is thus significant.

\section{REFERENCES}

1. W. Beenakker, R. Höpker, M. Spira, and P. M. Zerwas, Nucl. Phys. B492, 51-103 (1997), hep-ph/9610490.

2. W. Beenakker, M. Krämer, T. Plehn, M. Spira, and P. M. Zerwas, Nucl. Phys. B515, 3-14 (1998), hep-ph/9710451.

3. W. Hollik, M. Kollar, and M. K. Trenkel, JHEP 02, 018 (2008), 0712.0287.

4. M. Beccaria, G. Macorini, L. Panizzi, F. M. Renard, and C. Verzegnassi (2008), 0804.1252.

5. W. Hollik, and E. Mirabella (2008), 0806.1433.

6. W. Hollik, E. Mirabella, and M. K. Trenkel (2008), 0810.1044

7. J. R. Ellis, and S. Rudaz, Phys. Lett. B128, 248 (1983).

8. J. A. Aguilar-Saavedra, et al., Eur. Phys. J. C46, 43-60 (2006), hep-ph/0511344.

9. S. Bornhauser, M. Drees, H. K. Dreiner, and J. S. Kim, Phys. Rev. D76, 095020 (2007), 0709.2544.

10. G. Bozzi, B. Fuks, B. Herrmann, and M. Klasen, Nucl. Phys. B787, 1-54 (2007), 0704.1826.

11. U. Baur, S. Keller, and D. Wackeroth, Phys. Rev. D59, 013002 (1999), hep-ph/9807417.

12. K. P. O. Diener, S. Dittmaier, and W. Hollik, Phys. Rev. D69, 073005 (2004), hep-ph/ 0310364.

13. B. C. Allanach, Comput. Phys. Commun. 143, 305-331 (2002), hep-ph/0104145.

14. A. D. Martin, R. G. Roberts, W. J. Stirling, and R. S. Thorne, Eur. Phys. J. C39, 155-161 (2005), hep-ph/0411040. 